\title{
Tiprotec preserves endothelial function after cold ischemia and warm reperfusion: comparison between Saline, Custodiol and Tiprotec
}

\author{
Veres Gábor ${ }^{1,2^{*}}$, Hegedűs Péter ${ }^{1,2}$, Harald Schmidt ${ }^{1}$, Tamás Radovits², Raphael Zöller ${ }^{1}$, Matthias Karck',
} Gábor Szabó

From World Society of Cardiothoracic Surgeons 25th Anniversary Congress, Edinburgh

Edinburgh, UK. 19-22 September 2015

\section{Background/Introduction}

Background: Coronary artery bypass surgery provides excellent patency rates, however the early/late graft failure reduces the long-term benefit of myocardial revascularization.

\section{Aims/Objectives}

We investigated the effectiveness of generally used Saline, Custodiol solutions and a new solution (Tiprotec) at preserving endothelium after cold ischemia and warm reperfusion injury.

\section{Method}

Aortic transplantations were performed in Lewis rats. Aortic arches stored in Saline, Custodiol and Tiprotec solutions for 2 hours, then were transplanted into abdominal aorta. Two, 24 hours and 1 week after transplantation, the implanted grafts were harvested. Endothelium-dependent and-independent vasorelaxations were investigated in organ bath. DNA strand breaks were assessed by TUNEL-method, mRNA expressions by quantitative real-time PCR and the expression of CD-31 and $\alpha$-SMA by immunochemistry.

\section{Results}

Severely impaired endothelial function and integrity of implanted aortic grafts were shown after $2 \mathrm{~h}$ in the Saline, Custodiol group (maximal vasorelaxation to acetylcholine: control: $91 \pm 2 \%$, Saline: $26 \pm 5 \%$, Custodiol: $24 \pm 5 \%$, CD31 positive area control:96 $\pm 2 \%$, Saline: $35 \pm 13 \%$ Custodiol:54 $\pm 5 \%, \mathrm{p}<0.05$, respectively), however a preserved endothelial function was observed in the Tiprotec group when compared to the Saline and Custodiol group (maximal vasorelaxation: $46 \pm 7 \%$, CD31 positive area: $54 \pm 10 \%, \mathrm{p}<0.05)$. After 1 week, endothelial function were partially recovered in all groups, however it was significantly better in the Tiprotec group (maximal vasorelaxation to acetylcholine: Saline: $42 \pm 3 \%$, Custodiol:48 $\pm 3 \%$, Tiprotec: $56 \pm 3 \%$, CD31 positive area: Saline: $56 \pm 5 \%$, Custodiol: $54 \pm 4 \%$; Tiprotec: $83 \pm 6 \%$, $\mathrm{p}<$ 0.05 , respectively). In addition, mRNA levels of Bax, Bcl-2, eNOS, VEGF-2 and caspase-3 were significantly altered in both groups.

\section{Discussion/Conclusion}

Tiprotec appears to be superior for the preservation of endothelial- and smooth muscle cells of bypass graft after cold storage and warm reperfusion in our murine model.

\section{Authors' details}

'Department of Cardiac Surgery, University of Heidelberg, Heidelberg, 69120, Germany. ${ }^{2}$ Heart and Vascular Center, Semmelweis University, Budapest, 1220 , Hungary

Published: 16 December 2015

\section{doi:10.1186/1749-8090-10-S1-A74}

Cite this article as: Gábor et al:: Tiprotec preserves endothelial function after cold ischemia and warm reperfusion: comparison between Saline, Custodiol and Tiprotec. Journal of Cardiothoracic Surgery 2015 10(Suppl 1): A74. 